\title{
ON 3-DIMENSIONAL NORMAL ALMOST CONTACT METRIC MANIFOLDS SATISFYING CERTAIN CURVATURE CONDITIONS
}

\author{
Uday Chand De and Abul Kalam Mondal
}

\begin{abstract}
The object of the present paper is to study 3-dimensional normal almost contact metric manifolds satisfying certain curvature conditions. Among others it is proved that a parallel symmetric $(0,2)$ tensor field in a 3-dimensional non-cosympletic normal almost contact metric manifold is a constant multiple of the associated metric tensor and there does not exist a non-zero parallel 2-form. Also we obtain some equivalent conditions on a 3-dimensional normal almost contact metric manifold and we prove that if a 3-dimensional normal almost contact metric manifold which is not a $\beta$-Sasakian manifold satisfies cyclic parallel Ricci tensor, then the manifold is a manifold of constant curvature. Finally we prove the existence of such a manifold by a concrete example.
\end{abstract}

\section{Introduction}

Let $M$ be an almost contact manifold and $(\phi, \xi, \eta)$ its almost contact structure. This means, $M$ is an odd-dimensional differentiable manifold and $\phi, \xi, \eta$ are tensor fields on $M$ of types $(1,1),(1,0),(0,1)$ respectively, such that

$$
\phi^{2}=-I+\eta \otimes \xi, \quad \eta(\xi)=1 .
$$

Then also

$$
\phi \xi=0, \quad \eta \circ \phi=0 .
$$

Let $\mathbb{R}$ be the real line and $t$ a coordinate on $\mathbb{R}$. Define an almost complex structure $J$ on $M \times \mathbb{R}$ by

$$
J\left(X, \frac{\lambda d}{d t}\right)=\left(\phi X-\lambda \xi, \eta(X) \frac{d}{d t}\right),
$$

where the pair $(X, \lambda d / d t)$ denotes a tangent vector to $M \times \mathbb{R}, X$ and $\lambda d / d t$ being tangent to $M$ and $\mathbb{R}$ respectively.

$M$ and $(\phi, \xi, \eta)$ are said to be normal if the structure $J$ is integrable [1], [2].

Received September 9, 2008; Revised December 8, 2008.

2000 Mathematics Subject Classification. 53C15, 53C40.

Key words and phrases. normal almost contact metric manifolds, non-cosympletic, cyclic parallel Ricci tensor, Einstein manifold. 
The necessary and sufficient condition for $(\phi, \xi, \eta)$ to be normal is

$$
[\phi, \phi]+2 d \eta \otimes \xi=0
$$

where the pair $[\phi, \phi]$ is the Nijenhuis tensor of $\phi$ defined by

$$
[\phi, \phi](X, Y)=[\phi X, \phi Y]+\phi^{2}[X, Y]-\phi[\phi X, Y]-\phi[X, \phi Y]
$$

for any $X, Y \in \chi(M) ; \chi(M)$ being the Lie algebra of vector fields on $M$.

We say that the form $\eta$ has rank $r=2 s$ if $(d \eta)^{s} \neq 0$, and $\eta \wedge(d \eta)^{s}=0$, and has rank $r=2 s+1$ if $\eta \wedge(d \eta)^{S} \neq 0$ and $(d \eta)^{s+1}=0$. We also say that $r$ is the rank of the structure $(\phi, \xi, \eta)$.

A Riemannian metric $g$ on $M$ satisfying the condition

$$
g(\phi X, \phi Y)=g(X, Y)-\eta(X) \eta(Y)
$$

for any $X, Y \in \chi(M)$, is said to be compatible with the structure $(\phi, \xi, \eta)$. If $g$ is such a metric, then the quadruple $(\phi, \xi, \eta, g)$ is called an almost contact metric structure on $M$ and $M$ is an almost contact metric manifold. On such a manifold we also have

$$
\eta(X)=g(X, \xi)
$$

for any $X \in \chi(M)$ and we can always define the 2 -form $\Phi$ by

$$
\Phi(X, Y)=g(X, \phi Y)
$$

where $X, Y \in \chi(M)$.

It is no hard to see that if $\operatorname{dim} M=3$, then two Riemannian metric $g$ and $g$ are compatible with the same almost contact structure $(\phi, \xi, \eta)$ on $M$ if and only if

$$
\dot{g}=\sigma g+(1-\sigma) \eta \otimes \eta
$$

for a certain positive function $\sigma$ on $M$.

A normal almost contact metric structure $(\phi, \xi, \eta, g)$ satisfying additionally the condition $d \eta=\Phi$ is called Sasakian. Of course, any such structure on $M$ has rank 3. Also a normal almost contact metric structure satisfying the condition $d \Phi=0$ is said to be quasi Sasakian [3].

In a recent paper [8], Olszak studied the curvature properties of normal almost contact manifold of dimension three with several examples.

A Riemannian manifold is called Ricci-semisymmetric if

$$
R(X, Y) . S=0,
$$

where $R(X, Y)$ is treated as a derivation of the tensor algebra for any tangent vectors $X, Y ; R$ denotes the curvature tensor and $S$ is the Ricci tensor of type $(0,2)$ of the manifold.

Throughout this paper we consider $\alpha, \beta$ as constants.

In the present paper after preliminaries in Section 2 we prove in Section 3 that a parallel symmetric $(0,2)$ tensor field in a 3-dimensional non-cosympletic normal almost contact metric manifold is a constant multiple of the associated metric tensor and a parallel 2-form does not exist on such manifolds. In 
Section 4 for a Ricci-semisymmetric manifold we obtain some equivalent conditions. In the next section we prove that a 3 -dimensional normal almost contact manifold which is not a $\beta$-Sasakian manifold satisfying cyclic parallel Ricci tensor is a manifold of constant curvature. Finally we construct an example of a 3 -dimensional normal almost contact metric manifold which is not a $\beta$-Sasakian manifold.

\section{Preliminaries}

For a normal almost contact metric structure $(\phi, \xi, \eta, g)$ on $M$, we have [8]

$$
\begin{gathered}
\left(\nabla_{X} \phi\right)(Y)=g\left(\phi \nabla_{X} \xi, Y\right) \xi-\eta(Y) \phi \nabla_{X} \xi \\
\nabla_{X} \xi=\alpha\{X-\eta(X) \xi\}-\beta \phi X
\end{gathered}
$$

where $2 \alpha=\operatorname{div} \xi$ and $2 \beta=\operatorname{tr}(\phi \nabla \xi)$, $\operatorname{div} \xi$ is the divergence of $\xi$ defined by $\operatorname{div} \xi=\operatorname{trace}\left\{X \longrightarrow \nabla_{X} \xi\right\}$ and $\operatorname{tr}(\phi \nabla \xi)=\operatorname{trace}\left\{X \longrightarrow \phi \nabla_{X} \xi\right\}$.

(2.3) $R(X, Y) \xi=\left\{Y \alpha+\left(\alpha^{2}-\beta^{2}\right) \eta(Y)\right\} \phi^{2} X-\left\{X \alpha+\left(\alpha^{2}-\beta^{2}\right) \eta(X)\right\} \phi^{2} Y$

$$
+\{Y \beta+2 \alpha \beta \eta(Y)\} \phi X-\{X \beta+2 \alpha \beta \eta(X)\} \phi Y,
$$

$$
S(Y, \xi)=-Y \alpha-(\phi Y) \beta-\left\{\xi \alpha+2\left(\alpha^{2}-\beta^{2}\right)\right\} \eta(Y),
$$

$$
\xi \beta+2 \alpha \beta=0,
$$

where $R$ denotes the curvature tensor and $S$ is the Ricci tensor.

On the other hand, the curvature tensor in a 3-dimensional Riemannian manifold always satisfies

$$
\begin{aligned}
\tilde{R}(X, Y, Z, W)= & g(X, W) S(Y, Z)-g(X, Z) S(Y, W) \\
& +g(Y, Z) S(X, W)-g(Y, W) S(X, Z) \\
& -\frac{r}{2}[g(X, W) g(Y, Z)-g(X, Z) g(Y, W)],
\end{aligned}
$$

where $\tilde{R}(X, Y, Z, W)=g(R(X, Y) Z, W)$ and $r$ is the scalar curvature.

From $(2.3)$ we can derive that

$$
\tilde{R}(\xi, Y, Z, \xi)=-\left(\xi \alpha+\alpha^{2}-\beta^{2}\right) g(\phi Y, \phi Z)-(\xi \beta+2 \alpha \beta) g(Y, \phi Z) .
$$

By (2.4), (2.6) and (2.7) we obtain for $\alpha=$ constant and $\beta=$ constant,

$$
S(Y, Z)=\left(\frac{r}{2}+\alpha^{2}-\beta^{2}\right) g(\phi Y, \phi Z)-2\left(\alpha^{2}-\beta^{2}\right) \eta(Y) \eta(Z) .
$$

Applying (2.8) in (2.6) we get

$$
\begin{aligned}
R(X, Y) Z= & \left(\frac{r}{2}+2\left(\alpha^{2}-\beta^{2}\right)\right)\{g(Y, Z) X-g(X, Z) Y\} \\
& +g(X, Z)\left\{\left(\frac{r}{2}+3\left(\alpha^{2}-\beta^{2}\right)\right) \eta(Y) \xi\right\} \\
& -\left\{\frac{r}{2}+3\left(\alpha^{2}-\beta^{2}\right)\right\} \eta(Y) \eta(Z) X
\end{aligned}
$$




$$
\begin{aligned}
& -g(Y, Z)\left\{\left(\frac{r}{2}+3\left(\alpha^{2}-\beta^{2}\right)\right) \eta(X) \xi\right\} \\
& +\left(\frac{r}{2}+3\left(\alpha^{2}-\beta^{2}\right)\right) \eta(X) \eta(Z) Y .
\end{aligned}
$$

It is to be noted that the general formulas can be obtained by straightforward calculation.

From (2.5) it follows that if $\alpha, \beta=$ constant, then the manifold is either $\beta$ Sasakian, or $\alpha$-Kenmotsu [6] or cosympletic [1].

Proposition 1. A 3-dimensional normal almost contact metric manifold with $\alpha, \beta=$ constant is either $\beta$-Sasakian, or $\alpha$-Kenmotsu or cosympletic.

Definition 1. An almost $C(\lambda)$-manifold $M$ is an almost co-Hermitian manifold such that the Riemannian curvature tensor satisfies the following property: there exist $\lambda \in R$ such that for all $X, Y, Z, W \in \chi(M)$ :

$$
\begin{aligned}
R(X, Y, Z, W)= & R(X, Y, \phi Z, \phi W)+\lambda\{-g(X, Z) g(Y, W)+g(X, W) g(Y, Z) \\
& +g(X, \phi Z) g(Y, \phi W)-g(X, \phi W) g(Y, \phi Z)\} .
\end{aligned}
$$

A normal almost $C(\lambda)$-manifold is a $C(\lambda)$-manifold. If we take $\lambda=-\alpha^{2}$ for $\alpha>0$, then we get $C\left(-\alpha^{2}\right)$-manifold.

We note that $\beta$-Sasakian manifold are quasi-Sasakian [3]. They provide examples of $C(\lambda)$-manifolds with $\lambda \geq 0$.

An $\alpha$-Kenmotsu manifold is a $C\left(-\alpha^{2}\right)$-manifold [6].

Cosympletic manifolds provide a natural setting for time dependent mechanical systems as they are locally product of a Kaehler manifold and a real line or a circle [4].

\section{Second order parallel tensor field}

Let us consider a parallel symmetric $(0,2)$-tensor field $\delta$ on a 3 -dimensional normal almost contact metric manifold $M$.

Then, by $\nabla \delta=0$, we have

$$
\delta(R(U, V) X, Y)+\delta(X, R(U, V) Y)=0,
$$

where $U, V, X$ and $Y$ are arbitrary vectors fields on $M$.

As $\delta$ is symmetric, putting $U=X=Y=\xi$ in (3.1), we obtain

$$
\delta(\xi, R(\xi, X) \xi)=0 .
$$

Let us assume that $M$ is non-cosympletic. Take a non-empty connected open subset $U$ of $M$ and restrict our considerations to this set.

Now applying (2.3) in (3.2) we have

$$
\left(\alpha^{2}-\beta^{2}\right) \delta(X, \xi)-\left(\alpha^{2}-\beta^{2}\right) \eta(X) \delta(\xi, \xi)-2 \alpha \beta \delta(\phi X, \xi)=0 .
$$

Putting $\phi X$ instead of $X$ in (3.3) and using (1.1) we get

$$
\left(\alpha^{2}-\beta^{2}\right)^{2}\{\delta(X, \xi)-\eta(X) \delta(\xi, \xi)\}=0 .
$$


Since $M$ is non-cosympletic, we have

$$
\delta(X, \xi)-\eta(X) \delta(\xi, \xi)=0 .
$$
find

Differentiating (3.4) covariantly along $Y$ and applying (3.4) and (2.2) we

$$
\alpha\{\delta(X, Y)-\delta(\xi, \xi) g(X, Y)\}=\beta\{\delta(X, \phi Y)-\delta(\xi, \xi) g(X, \phi Y)\} .
$$

Putting $\phi Y$ instead of $Y$ in (3.5) and using (1.1) we have

$$
\left(\alpha^{2}+\beta^{2}\right)\{\delta(X, Y)-\delta(\xi, \xi) g(X, Y)\}=0
$$

This implies

$$
\delta(X, Y)=\delta(\xi, \xi) g(X, Y), \quad \text { since } \quad \alpha^{2}+\beta^{2} \neq 0 .
$$

Hence, since $\delta$ and $g$ are parallel tensor fields, $\lambda=\delta(\xi, \xi)$ is constant on $U$. By the parallelity of $\delta$ and $g$ it must be $\delta=\lambda g$ on whole of $M$. Thus we have the following:

Theorem 3.1. A parallel symmetric $(0,2)$ tensor field in a 3-dimensional noncosympletic normal almost contact metric manifold is a constant multiple of the associated metric tensor.

As an immediate corollary of Theorem 3.1 we have the following result:

Corollary 3.1. If the Ricci tensor field in a 3-dimensional normal almost contact metric manifold is parallel, then it is an Einstein manifold.

Let us now assume that $\delta$ is a parallel 2 -form on $M$, that is, $\delta(X, Y)=$ $-\delta(Y, X)$ and $\nabla \delta=0$.

Then

$$
\delta(\xi, \xi)=0 .
$$

Covariant differentiation of (3.7) implies

$$
\delta\left(\nabla_{X} \xi, \xi\right)=0 .
$$

By (2.2) and (3.7) we obtain from (3.8)

$$
\alpha \delta(X, \xi)-\beta \delta(\phi X, \xi)=0 .
$$

Putting $\phi X$ instead of $X$ in (3.9) and using (1.1) we have

$$
\left(\alpha^{2}+\beta^{2}\right) \delta(X, \xi)=0 .
$$

Assume the manifold $M$ is non-cosympletic and consider a non-empty open subset $U$ of $M$. Then on $U$ we have

$$
\delta(X, \xi)=0 .
$$

Covariant differentiation of the above and using (2.2) and (3.11) gives

$$
\alpha \delta(X, Y)-\beta \delta(X, \phi Y)=0 .
$$


Putting $\phi Y$ instead of $Y$ in (3.12) and using (1.1) we get

$$
\left(\alpha^{2}+\beta^{2}\right) \delta(X, Y)=0 \text {. }
$$

Since $\alpha^{2}+\beta^{2} \neq 0$, this implies

$$
\delta(X, Y)=0 .
$$

Hence $\delta=0$ on $U$. Since $\delta$ is parallel on $U, \delta=0$ on $M$.

Thus we have the following:

Theorem 3.2. On a 3-dimensional non-cosympletic normal almost contact metric manifold there does not exist a non-zero parallel 2-form.

\section{Ricci-semisymmetric normal almost contact metric manifold}

Let us consider a 3-dimensional normal almost contact metric manifold which satisfies the condition

$$
R(X, Y) . S=0
$$

for any $X, Y \in \chi(M)$.

Then we have

$$
S(R(X, Y) U, V)+S(U, R(X, Y) V)=0 .
$$

Putting $X=U=\xi$ in (4.1), we have

$$
S(R(\xi, Y) \xi, V)+S(\xi, R(\xi, Y) V)=0 .
$$

Using (2.3) in (4.2), we have

(4.3) $\left(\alpha^{2}-\beta^{2}\right)\{S(Y, V)-\eta(Y) S(\xi, V)+\eta(V) S(\xi, Y)-g(Y, V) S(\xi, \xi)\}=0$.

Let us assume that $M$ is non-cosympletic. Take a nonempty connected open subset $U$ of $M$ and restrict our considerations to this set. Then from (4.3) we have

$$
S(Y, V)-\eta(Y) S(\xi, V)+\eta(V) S(\xi, Y)-g(Y, V) S(\xi, \xi)=0 .
$$

Now using (2.4) in (4.4) we get

$$
S(Y, V)-S(\xi, \xi) g(Y, V)+\eta(Y)(\phi V) \beta-\eta(V) \phi(Y) \beta=0 .
$$

Again putting $U=V=\xi$ in (4.1) we have

$$
S(\xi, R(X, Y) \xi)=0 .
$$

Applying (2.3) in (4.6) we have

$$
\begin{aligned}
& \left(\alpha^{2}-\beta^{2}\right)\{\eta(X) S(\xi, Y)-\eta(Y) S(\xi, X)\} \\
= & 2 \alpha \beta\{\eta(X) S(\xi, \phi Y)-\eta(Y) S(\xi, \phi X)\} .
\end{aligned}
$$

Using (2.4) in (4.7) we get

$$
\left(\alpha^{2}-\beta^{2}\right)\{\eta(X)(\phi Y) \beta-\eta(Y)(\phi X) \beta\}=0
$$


which implies that, since $\alpha^{2}-\beta^{2} \neq 0$,

$$
\eta(X)(\phi Y) \beta=\eta(Y)(\phi X) \beta
$$

on $M$.

Now using (4.9) in (4.5) we get

$$
S(Y, V)=S(\xi, \xi) g(Y, V) .
$$

Clearly from (2.4) it follows

$$
S(\xi, \xi)=2\left(\beta^{2}-\alpha^{2}\right)
$$

Therefore from (4.10) we obtain

$$
S(Y, V)=2\left(\beta^{2}-\alpha^{2}\right) g(Y, V),
$$

which implies that $M$ is an Einstein manifold with constant curvature $6\left(\beta^{2}-\right.$ $\left.\alpha^{2}\right)$. So we have the following:

Theorem 4.1. Let $M$ be a 3-dimensional non-cosympletic normal almost contact metric manifold. Then the following conditions are equivalent:

(i) $M$ is an Einstein manifold;

(ii) The Ricci tensor $S$ of $M$ is parallel, i.e., $\nabla S=0$;

(iii) $M$ is Ricci-semisymmetric.

Remark. It is obvious that by the formula (2.6) the conditions (i), (ii), and (iii) in Theorem 4.1 can be replaced by the following conditions:

(i) $M$ is of constant curvature;

(ii) $M$ is locally symmetric $(\nabla R=0)$;

(iii) $M$ is semisymmetric $(R \cdot R=0)$.

5. 3-dimensional normal almost contact metric manifold satisfying cyclic parallel Ricci tensor

A. Gray [5] introduced two classes of Riemannian manifold determined by covariant derivative of Ricci tensor. The class $A$ consisting of all Riemannian manifold whose Ricci tensor $S$ is a Codazzi tensor, i.e.,

$$
\left(\nabla_{X} S\right)(Y, Z)=\left(\nabla_{Y} S\right)(X, Z) .
$$

The class $B$ consisting of all Riemannian manifolds whose Ricci tensor is cyclic parallel, i.e.,

$$
\left(\nabla_{X} S\right)(Y, Z)+\left(\nabla_{Y} S\right)(X, Z)+\left(\nabla_{Z} S\right)(X, Y)=0 .
$$

A Riemannian manifold is said to satisfy cyclic parallel Ricci tensor if the Ricci tensor is non-zero and satisfies the condition (5.1). It is known [7] that Cartan hypersurface are manifolds with non-parallel Ricci tensor satisfying the condition (5.1).

From (5.1) it follows that $r=$ constant. Hence from (2.8), using (1.5) we have

$$
\left(\nabla_{X} S\right)(Y, Z)=-\left(\frac{r}{2}+3\left(\alpha^{2}-\beta^{2}\right)\right)\left\{\eta(Y)\left(\nabla_{X} \eta\right) Z+\eta(Z)\left(\nabla_{X} \eta\right) Y\right\}
$$


Applying (5.2) in (5.1) we have

$$
\begin{aligned}
\left(\frac{r}{2}+3\left(\alpha^{2}-\beta^{2}\right)\right)\{ & \eta(Y)\left(\nabla_{X} \eta\right) Z+\eta(Z)\left(\nabla_{X} \eta\right) Y \\
& +\eta(X)\left(\nabla_{Y} \eta\right) Z+\eta(Z)\left(\nabla_{Y} \eta\right) X \\
& \left.+\eta(X)\left(\nabla_{Z} \eta\right) Y+\eta(Y)\left(\nabla_{Z} \eta\right) X\right\}=0
\end{aligned}
$$

Using (2.2) and putting $Y=Z=e_{i}$, where $\left\{e_{i}\right\}$ is an orthonormal basis of the tangent space at each point of the manifold, in (5.3) we get

$$
\left(\frac{r}{2}+3\left(\alpha^{2}-\beta^{2}\right)\right) 2 \alpha \eta(X)=0 .
$$

This implies either

$$
\alpha=0,
$$

which gives the manifold is $\beta$-Sasakian manifold. Or,

$$
\left(\frac{r}{2}+3\left(\alpha^{2}-\beta^{2}\right)\right) \eta(X)=0
$$

which gives

$$
r=6\left(\beta^{2}-\alpha^{2}\right)
$$

Conversely, if $r=6\left(\beta^{2}-\alpha^{2}\right)$, then from $(5.2)$ it follows that $\left(\nabla_{X} S\right)(Y, Z)=0$ and hence the manifold satisfies cyclic parallel Ricci tensor.

This leads to the following lemma:

Lemma 5.1. A 3-dimensional normal almost contact metric manifold which is not a $\beta$-Sasakian manifold satisfies cyclic parallel Ricci tensor if and only if $r=6\left(\beta^{2}-\alpha^{2}\right)$.

From (2.8) we have

$$
S(Y, Z)=\left(\frac{r}{2}+\alpha^{2}-\beta^{2}\right) g(Y, Z)-\left(\frac{r}{2}+3\left(\alpha^{2}-\beta^{2}\right)\right) \eta(Y) \eta(Z),
$$

which implies that

$$
Q(Y)=\left(\frac{r}{2}+\alpha^{2}-\beta^{2}\right) Y-\left(\frac{r}{2}+3\left(\alpha^{2}-\beta^{2}\right)\right) \eta(Y) \xi
$$

where $Q$ is the symmetric endomorphism of the tangent space at each point of the manifold corresponding to the Ricci tensor $S$, i.e., $S(X, Y)=g(Q X, Y)$.

Using (5.5) and (5.6) from (2.6) we get

$$
\begin{aligned}
R(X, Y) Z= & \left(3 \frac{r}{2}+2\left(\alpha^{2}-\beta^{2}\right)\right)\{g(Y, Z) X-g(X, Z) Y\} \\
& -\left(\frac{r}{2}+3\left(\alpha^{2}-\beta^{2}\right)\right)\{g(Y, Z) \eta(X) \xi-g(X, Z) \eta(Y) \xi \\
& +\eta(Y) \eta(Z) X-\eta(X) \eta(Z) Y\} .
\end{aligned}
$$

From (5.7) it is clear that if $r=6\left(\beta^{2}-\alpha^{2}\right)$, then the manifold is a manifold of constant curvature.

This leads by virtue of Lemma 5.1. to the following theorem: 
Theorem 5.1. If a 3-dimensional normal almost contact metric manifold which is not a $\beta$-Sasakian manifold satisfying cyclic parallel Ricci tensor, then the manifold is a manifold of constant curvature $6\left(\beta^{2}-\alpha^{2}\right)$.

\section{Example of a 3-dimensional normal almost contact metric manifold}

We consider the 3 -dimensional manifold $M=\left\{(x, y, z) \in \mathbb{R}^{3}, z \neq 0\right\}$, where $(x, y, z)$ are standard co-ordinate of $\mathbb{R}^{3}$.

The vector fields

$$
e_{1}=z \frac{\partial}{\partial x}, \quad e_{2}=z \frac{\partial}{\partial y}, \quad e_{3}=z \frac{\partial}{\partial z}
$$

are linearly independent at each point of $M$.

Let $g$ be the Riemannian metric defined by

$$
\begin{aligned}
& g\left(e_{1}, e_{3}\right)=g\left(e_{1}, e_{2}\right)=g\left(e_{2}, e_{3}\right)=0, \\
& g\left(e_{1}, e_{1}\right)=g\left(e_{2}, e_{2}\right)=g\left(e_{3}, e_{3}\right)=1
\end{aligned}
$$

that is, the form of the metric becomes

$$
g=\frac{d x^{2}+d y^{2}+d z^{2}}{z^{2}}
$$

Let $\eta$ be the 1 -form defined by $\eta(Z)=g\left(Z, e_{3}\right)$ for any $Z \in \chi(M)$.

Let $\phi$ be the $(1,1)$ tensor field defined by

$$
\phi\left(e_{1}\right)=-e_{2}, \quad \phi\left(e_{2}\right)=e_{1}, \quad \phi\left(e_{3}\right)=0 .
$$

Then using the linearity of $\phi$ and $g$, we have

$$
\begin{gathered}
\eta\left(e_{3}\right)=1, \\
\phi^{2} Z=-Z+\eta(Z) e_{3}, \\
g(\phi Z, \phi W)=g(Z, W)-\eta(Z) \eta(W)
\end{gathered}
$$

for any $Z, W \in \chi(M)$.

Then for $e_{3}=\xi$, the structure $(\phi, \xi, \eta, g)$ defines an almost contact metric structure on $M$.

Let $\nabla$ be the Levi-Civita connection with respect to metric $g$. Then we have

$$
\begin{aligned}
{\left[e_{1}, e_{3}\right] } & =e_{1} e_{3}-e_{3} e_{1} \\
& =z \frac{\partial}{\partial x}\left(z \frac{\partial}{\partial z}\right)-z \frac{\partial}{\partial z}\left(z \frac{\partial}{\partial x}\right) \\
& =z^{2} \frac{\partial^{2}}{\partial x \partial z}-z^{2} \frac{\partial^{2}}{\partial z \partial x}-z \frac{\partial}{\partial x} \\
& =-e_{1} .
\end{aligned}
$$

Similarly

$$
\left[e_{1}, e_{2}\right]=0 \text { and }\left[e_{2}, e_{3}\right]=-e_{2} \text {. }
$$


The Riemannian connection $\nabla$ of the metric $g$ is given by

$$
\begin{aligned}
2 g\left(\nabla_{X} Y, Z\right)= & X g(Y, Z)+Y g(Z, X)-Z g(X, Y) \\
& -g(X,[Y, Z])-g(Y,[X, Z])+g(Z,[X, Y]),
\end{aligned}
$$

which known as Koszul's formula.

Using (6.1) we have

$$
\begin{aligned}
2 g\left(\nabla_{e_{1}} e_{3}, e_{1}\right) & =-2 g\left(e_{1}, e_{1}\right) \\
& =2 g\left(-e_{1}, e_{1}\right) .
\end{aligned}
$$

Again by (6.1)

$$
2 g\left(\nabla_{e_{1}} e_{3}, e_{2}\right)=0=2 g\left(-e_{1}, e_{2}\right)
$$

and

$$
2 g\left(\nabla_{e_{1}} e_{3}, e_{3}\right)=0=2 g\left(-e_{1}, e_{3}\right) .
$$

From (6.2), (6.3) and (6.4) we obtain

$$
2 g\left(\nabla_{e_{1}} e_{3}, X\right)=2 g\left(-e_{1}, X\right)
$$

for all $X \in \chi(M)$.

Thus

$$
\nabla_{e_{1}} e_{3}=-e_{1} .
$$

Therefore, (6.1) further yields

$$
\begin{gathered}
\nabla_{e_{1}} e_{3}=-e_{1}, \quad \nabla_{e_{1}} e_{2}=0, \quad \nabla_{e_{1}} e_{1}=e_{3} \\
\nabla_{e_{2}} e_{3}=-e_{2}, \quad \nabla_{e_{2}} e_{2}=e_{3}, \quad \nabla_{e_{2}} e_{1}=0 \\
\nabla_{e_{3}} e_{3}=0, \quad \nabla_{e_{3}} e_{2}=0, \quad \nabla_{e_{3}} e_{1}=0 .
\end{gathered}
$$

(6.5) tells us that the manifold satisfies (2.2) for $\alpha=-1$ and $\beta=0$ and $\xi=e_{3}$. Hence the manifold is a normal almost contact metric manifold with $\alpha, \beta=$ constants.

It is known that

$$
R(X, Y) Z=\nabla_{X} \nabla_{Y} Z-\nabla_{Y} \nabla_{X} Z-\nabla_{[X, Y]} Z .
$$

With the help of the above results and using (6.6) it can be easily verified that

$$
\begin{gathered}
R\left(e_{1}, e_{2}\right) e_{3}=0, \quad R\left(e_{2}, e_{3}\right) e_{3}=-e_{2}, \quad R\left(e_{1}, e_{3}\right) e_{3}=-e_{1}, \\
R\left(e_{1}, e_{2}\right) e_{2}=-e_{1}, \quad R\left(e_{2}, e_{3}\right) e_{2}=e_{3}, \quad R\left(e_{1}, e_{3}\right) e_{2}=0, \\
R\left(e_{1}, e_{2}\right) e_{1}=e_{2}, \quad R\left(e_{2}, e_{3}\right) e_{1}=0, \quad R\left(e_{1}, e_{3}\right) e_{1}=e_{3} .
\end{gathered}
$$

From the above expressions of the curvature tensor we obtain

$$
\begin{aligned}
S\left(e_{1}, e_{1}\right) & =g\left(R\left(e_{1}, e_{2}\right) e_{2}, e_{1}\right)+g\left(R\left(e_{1}, e_{3}\right) e_{3}, e_{1}\right) \\
& =-2 .
\end{aligned}
$$

Similarly we have

$$
S\left(e_{2}, e_{2}\right)=S\left(e_{3}, e_{3}\right)=-2 .
$$


Therefore,

$$
r=S\left(e_{1}, e_{1}\right)+S\left(e_{2}, e_{2}\right)+S\left(e_{3}, e_{3}\right)=-6 .
$$

We note that here $\alpha, \beta$ and $r$ are all constants.

We claim that $M$ with the given metric $g$, is a Ricci-semisymmetric normal almost contact metric manifold.

To verify the relation (4.11) it is sufficient to check

$$
S\left(e_{i}, e_{i}\right)=-2=-2\left(\alpha^{2}-\beta^{2}\right) g\left(e_{i}, e_{i}\right)
$$

for all $i=1,2,3$ and $\alpha=-1, \beta=0$. Hence $M$ is an Einstein manifold.

Also the manifold satisfies cyclic parallel Ricci tensor. $\alpha \neq 0$ implies that the manifold is not a $\beta$-Sasakian manifold. Since $r=-6=6\left(\beta^{2}-\alpha^{2}\right)$ for $\alpha=-1, \beta=0$, therefore Theorem 5.1 holds.

Acknowledgement. The authors are thankful to Prof. J. S. Pak of Kyungpook National University, Korea, for his comments and valuable suggestions towards the improvement of this paper.

\section{References}

[1] D. E. Blair, Contact Manifolds in Riemannian Geometry, Lecture Note in Mathematics, Vol. 509, Springer-Verlag, Berlin-New York, 1976.

[2] _ Riemannian geometry of contact and symplectic manifolds, Progress in Mathematics, Vol. 203, Birkhauser Boston, Inc., Boston, 2002.

[3] - The theory of quasi Sasakian structures, J. Differ. Geometry 1 (1976), 331-345.

[4] F. Cantrijnt, M. De Leon, and E. A. Lacomha, Gradient vector fields in cosympletic manifolds, J. Phys. A. Math. Gen. 25 (1992), 409-416.

[5] A. Gray, Einstein-like manifolds which are not Einstein, Geom. Dedicata 7 (1978), no. $3,259-280$.

[6] D. Janssen and L. Vanhecke, Almost contact structures and curvature tensors, Kodai Math. J. 4 (1981), 1-27.

[7] U. H. Ki and H. Nakagawa, A characterization of the Cartan hypersurface in a sphere, Tohoku Math. J. 39 (1987), 27-40.

[8] Z. Olszak, Normal almost contact manifolds of dimension three, Annales Pol. Math. XLVII (1986), 41-50.

UDAY Chand DE

Department of Mathematics

UNIVERSITY OF KALYANI

KALYANI-741235

West Bengal, India

E-mail address: uc_de@yahoo.com

Abul Kalam Mondal

DumDum Subhasnagar High School(H.S.)

43, Sarat Bose Road

KolKATA-700065

West Bengal, IndiA

E-mail address: kalam_ju@yahoo.co.in 